\title{
Evaluación de la efectividad de distintas formulaciones de jabón con extracto de Hypericum mexicanum L.
}

Evaluation of the effectiveness of different formulations of soap with extract of Hypericum mexicanum L.

\section{Avaliação da eficácia de diferentes formulações de sabão com extracto de Hipericão mexicanum $L$.}

\author{
Diana Carolina Corzo-Barragán ${ }^{1} \&$ Diana Milena Gaitán-Vaca² \\ ${ }^{1}$ Ingeniera Agroindustrial, Especialista en Gestión de Proyectos, Magister en Diseño de Productos y Procesos. \\ ${ }^{2}$ Microbióloga Industrial, Magister en Bioinformática. \\ 1,2Jardín Botánico de Bogotá José Celestino Mutis. \\ 1dianacorbarra1@gmail.com; 2dianitadark@hotmail.com
}

\section{Resumen}

Con el objetivo de evaluar la efectividad de distintas formulaciones de jabón líquido y sólido con extracto etanólico de Hypericum mexicanum L., se llevó a cabo una prueba de manipuladores. El recuento microbiano permitió analizar la efectividad en cada uno de los tratamientos evaluados, encontrando que el jabón líquido y sólido en concentración del 0,1\% de extracto es el más efectivo; sin embargo, no existen diferencias significativas entre la concentración de extracto $(0,1-0,2 \%)$ y el control (jabón comercial). Por otro lado, usando el método de difusión en disco, se evaluó la actividad antimicrobiana de los distintos tratamientos, frente a (Escherichia coli, Staphylococcus aureus, Staphylococcus epidermidis y Pseudomonas aeruginosa), con lo cual se determinó que: 1) el jabón líquido en concentración de $0,1 \%$ y $0,2 \%$ de extracto vegetal y el jabón comercial inhiben el crecimiento de Escherichia coli; 2) todos los tratamientos presentaron inhibición frente a Staphylococcus aureus, exceptuando el de uso comercial y el sólido con extracto vegetal al 0,1\%; 3) para Staphylococcus epidermidis, se presentó una inhibición del jabón sólido y líquido sin extracto y del jabón líquido con extracto vegetal al 0,2\%; 4) en la evaluación de Pseudomonas aeruginosa, se observó inhibición del jabón líquido con concentración de extracto del $0,1 \%$ y $0,2 \%$ y del jabón comercial.

Palabras clave: actividad antibacteriana, extracto vegetal, producto natural, prueba de manipuladores.

\begin{abstract}
To evaluate the effectiveness of different formulations of liquid and solid soap with ethanol extract of Hypericum mexicanum $\mathrm{L}$, it was carried out a test handler, the microbial count allowed to observe the effectiveness of each of the treatments evaluated, finding that liquid and solid concentration of $0,1 \%$ extract soap is the most effective, however, no
\end{abstract}


significant differences between the concentration of extract $(0.1-0.2 \%)$ and control (commercial soap). Furthermore, using the disk diffusion method, the antimicrobial activity of different treatments against (Escherichia coli, Staphylococcus aureus, Staphylococcus epidermidis and Pseudomonas aeruginosa) was evaluated, whereby it was determined that: 1) liquid soap in concentration of $0.1 \%$ and $0.2 \%$ plant extract and commercial soap inhibit the growth of Escherichia coli; 2) all treatments showed inhibition against Staphylococcus aureus, except for commercial use and solid vegetable extract $0.1 \%$; 3 ) for Staphylococcus epidermidis, inhibition of solid and liquid soap without extract and liquid soap with plant extract at $0,2 \%$ was introduced; 4 ) in the evaluation of Pseudomonas aeruginosa, liquid soap inhibition was observed with extract concentration of $0.1 \%$ and $0.2 \%$ commercial soap.

Key-words: antimicrobial activity, vegetable extract, natural product, test handler,

\section{Resumo}

Com o objetivo de avaliar a efetividade de diferentes formulações de sabonete líquido e sólido com extrato de etanol de Hypericum mexicanum L., foi feita um teste de manipulação. A contagem microbiana permitiu analisar a efetividade de cada um dos tratamentos avaliados, encontrando-se que o sabonete líquido e sólido em concentrações de $0,1 \%$ de extrato é o mais eficiente. No entanto, diferenças significativas não existem entre a concentração do extrato $(0,1-0,2 \%)$ e o controle (sabonete comercial). Utilizando-se o método de difusão em disco foi avaliada a atividade antimicrobiana dos diferentes tratamentos frente a (Escherichia coli, Staphylococcus aureus, Staphylococcus epidermidis e Pseudomonas aeruginosa), determinando-se: 1) o sabonete líquido nas concentrações de $0,1 \%$ e $0,2 \%$ de extrato vegetal e o sabonete comercial inibem o crescimento de Escherichia coli; 2) todos os tratamentos apresentaram inibição para Staphylococcus aureus, com exceção do uso comercial e o sólido com extrato vegetal ao 0,1\%; 3) para Staphylococcus epidermidis, foi observada inibição do sabonete sólido e líquido sem extrato e do sabonete líquido com extrato vegetal ao $0,2 \%$; 4) na avaliação de Pseudomonas aeruginosa, foi observada inibição do sabonete líquido na concentração do extrato de $0,1 \%$ e $0,2 \%$ e do sabonete comercial.

Palavras-chave: atividade antibacteriana, extrato vegetal, produto natural, teste de manipulação.

\section{Introducción}

Si bien Colombia tiene una alta diversidad de recursos vegetales, estos aún no han sido estudiados en su totalidad. En esta perspectiva, entidades como el Jardín Botánico de Bogotá -JBB- adelanta estudios de bioprospección orientados al desarrollo de investigaciones que conduzcan a la búsqueda de productos naturales con propiedades medicinales, industriales, farmacológicas y/o biotecnológicas. Los estudios de bioprospección involucran el conocimiento de la biodiversidad y pueden estar soportados en saberes tradicionales o indígenas (Melgarejo et al., 2002). Los resultados de este tipo de investigaciones han promovido el uso de productos farmacéuticos derivados de fuentes naturales, los cuales se han convertido en una alternativa para el tratamiento de enfermedades causadas por la proliferación de microorganismos patógenos. En este sentido y considerando la importancia que tiene hoy en día la búsqueda de fuentes naturales, las plantas son una opción para la obtención de compuestos bioactivos. Las investigaciones en este campo son cada vez más numerosas; así, en los últimos años, más de la mitad de los productos farmacéuticos son derivados de fuentes naturales (Newman \& Cragg, 2007).

En la región andina existen diversas especies nativas con potenciales aún no estudiados; una de estas especies es Hypericum mexicanum L., Ilamada comúnmente chite, en las zonas rurales del Distrito Capital de Bogotá. La decocción de hojas 
de Hypericum mexicanum L., es usada para tratar problemas renales o en baños para lavar heridas; igualmente, sus raíces, mezcladas con otras especies, se toman para aliviar dolores (Gutiérrez, Pineda, García, 2011). El uso etnobotánico del género Hypericum es conocido a nivel mundial; sus hojas y aceites esenciales son empleados para el tratamiento de diversas enfermedades. Varias especies de Hypericum han demostrado actividad antibacteriana contra microorganismos como Staphylococcus aureus, Bacillus cereus o Bacillus subtilis. Esto podría explicar el uso popular de algunas especies de Hypericum para la curación de heridas y el tratamiento de enfermedades infecciosa (Ishiguro, Yamaki, Kashihara, \& Takagi, 1986; Jayasuriya, McChesney, Swanson, 1989; Rocha et al., 1994; Yamaki \& Ishiguro, 1994). La especie más estudiada del género Hypericum es $H$. perforatum L., la cual ha sido objeto de varios estudios por su potencial biotecnológico; ensayos clínicos han validado su uso como antidepresivo, antiviral -antirretroviral, incluidos los efectos anti-VIH-, y actividades antibacterianas (Barnes, Anderson, \& Phillipson, 2001) including antidepressant, antiviral, and antibacterial effects, provide supporting evidence for several of the traditional uses stated for St John's wort. Many pharmacol. activities appear to be attributable to hypericin and to the flavonoid constituents; hypericin is also reported to be responsible for the photosensitive reactions that have been documented for St. John's wort. With regard to the antidepressant effects of St John's wort, hyperforin, rather than hypericin as originally thought, has emerged as one of the major constituents responsible for antidepressant activity. Further research is required to det. which other constituents contribute to the antidepressant effect. Evidence from randomized controlled trials has confirmed the efficacy of St John's wort exts. over placebo in the treatment of mild-tomoderately severe depression. Other randomized controlled studies have provided some evidence that St John's wort exts. are as effective as some std. antidepressants in mild-to-moderate depression. There is still a need for further trials to assess the efficacy of St John's wort exts., compared with that of std. antidepressants, particularly newer antidepressant agents, such as the selective serotonin reuptake inhibitors (recent comparative studies with fluoxetine and sertraline have been conducted. La actividad antibacteriana también se ha reportado para otras especies de Hypericum (Gibbons, 2008; Gibbons, Ohlendorf, \& Johnsen, 2002; Pistelli et al., 2000; Rabanal, Arias, Prado, Hernández-Pérez, \& Sánchez-Mateo, 2002); otros estudios califican al género Hypericum como fuente potencial antibacteriana (Gibbons, 2008). Estudios preliminares evidenciaron que $H$. mexicanum inhibe el crecimiento bacteriano de Escherichia coli, Pseudomonas aeruginosa y Staphylococcus aureus (Rodríguez, 2012).

Considerando el potencial de la especie, se elaboró un jabón desinfectante en presentación sólida y líquida, empleando como ingrediente activo el extracto etanólico de las hojas de Hypericum mexicanum L., en dos concentraciones, para posteriormente verificar la efectividad del producto terminado mediante la realización de la prueba de manipuladores, la cual permitió conocer la cantidad de microorganismos presentes antes y después del uso del producto; adicionalmente, los productos fueron probados, por medio de la técnica de difusión en disco, frente a cepas de referencia: Escherichia coli, Pseudomonas aeruginosa, Staphylococcus aureus y Staphylococcus epidermidis.

\section{Materiales y métodos}

Recolección de material vegetal y obtención de extracto: Se recolectaron hojas de Hypericum mexicanum $\mathrm{L}$. en la localidad de Sumapaz $\left(4^{\circ} 15^{\prime} 36^{\prime \prime} \mathrm{N} 74^{\circ} 10^{\prime} 42^{\prime \prime} \mathrm{O}\right)$; un espécimen para identificación y testigo fue colectado por el biólogo Gustavo Morales y reposa en el herbario del Jardín Botánico de Bogotá -identificado como GM 3550-. El extracto etanólico, fue obtenido por la línea de fitoquímica, siguiendo el siguiente procedimiento: se secaron las hojas a temperatura ambiente por 8 días; posteriormente se hizo una reducción de tamaño de forma mecánica y se sometió a maceración en frío usando etanol al 96\% en recipientes ámbar por 3 días; luego se filtró y el extracto etanólico resultante se concentró sucesivamente a presión reducida en un rotavaporador marca Heidolp; el extracto se 
almacenó hasta su uso en cabina de extracción de gases LEX C-4.

Formulación de producto desinfectante: Se formuló un producto desinfectante en presentación líquida y sólida usando base neutra de glicerina para el jabón sólido y texapón para el jabón líquido; en cada una de las presentaciones se empleó una concentración de extracto vegetal de $0,1 \%$ y $0,2 \%$, además de moldes, recipientes, balanza y vidriería de medición. Para la elaboración de jabón artesanal se siguió el procedimiento descrito por Westerman (Westerman, 2001). En total se evaluaron siete tratamientos: 1) jabón con $0 \%$ de extracto en presentación sólida; 2) jabón con $0 \%$ de extracto en presentación líquida; 3) jabón con $0,1 \%$ de extracto en presentación sólida; 4) jabón con $0,1 \%$ de extracto en presentación líquida; 5) jabón con 0,2 \% extracto en presentación sólida; 6) jabón con 0,2\% extracto en presentación líquida y 7) jabón comercial en presentación líquida -empleado como control-.

Efectividad del producto: la efectividad del producto fue evaluada por el personal técnico operativo de la Subdirección Científica del Jardín Botánico de Bogotá, mediante la realización de la prueba de manipuladores, la cual se hizo antes y después del lavado de manos -con cada uno de los siete tratamientos-, por medio de un frotis realizado con hisopos de algodón estéril en la mano predominante, para ello se adaptó la metodología de Alvarado, García \& Arias-echandi (2010). El hisopo se introdujo en solución salina $(0.85 \%$ p/v), para luego realizar la respectiva siembra en agar nutritivo + nistatina - para aislamiento de bacterias-. Las cajas se sembraron por separado mediante la técnica de agotamiento y se incubaron a $35 \pm 2^{\circ} \mathrm{C}$ por 24 horas (Castro, 2011). Transcurrido el tiempo de incubación, se llevó a cabo el recuento en placa y la identificación microscópica de las bacterias por medio de tinción de Gram. Los ensayos se realizaron por triplicado antes y después del uso de cada tratamiento. El lavado de manos se realizó teniendo en cuenta las siguientes características: se humedecieron las palmas de las manos con agua potable; se aplicó $1 \mathrm{ml}$ de jabón en el caso de la presentación líquida y 0.5 $\mathrm{g}$ de jabón en presentación sólida; se frotó por 30 segundos, se enjuagó con $200 \mathrm{ml}$ de agua potable y se secaron las manos con toallas de papel estéril; para verificar la pureza del medio y descartar contaminación se incubaron tres cajas Petri sin inocular.

\section{Evaluación de actividad antimicrobiana frente} a cepas de referencia: Se probaron los distintos tratamientos, frente a bacterias Gram negativas y Gram positivas (E. coli, ATCC 25922, P. aeruginosa, ATCC 9027, S. aureus ATCC 25923, S. epidermidis ATCC, 1228), procedentes de cepas de referencia obtenidas del cepario de la Universidad Javeriana Sede Bogotá; estas bacterias fueron seleccionadas por su importancia en la salud pública. Se empleó la técnica de difusión en pozo para evidenciar la inhibición de los distintos tratamientos; para ello se aislaron las bacterias en agar nutritivo, preparado según especificaciones de la casa matriz, por la técnica de agotamiento, se incubaron a $35 \pm 2^{\circ} \mathrm{C}$ por 24 horas Posteriormente, con ayuda de un asa redonda, se tomaron 2 a 5 colonias y se solubilizaron en solución salina estéril $(0,85 \% \mathrm{p} / \mathrm{v})$ hasta alcanzar la turbidez comparable con el patrón $\mathrm{N}^{\circ} 5$ de MacFarland. Para el ensayo de actividad antibacterial, se llevó a cabo una siembra masiva en agar nutritivo de $100 \mu \mathrm{l}$ del patrón de MacFarland, posteriormente se realizaron pozos de $0,5 \mathrm{~cm}$ de diámetro y se depositaron $50 \mu \mathrm{l}$ de cada tratamiento en cada pozo; como control positivo se usó cloranfenicol en concentración de 100 $\mu \mathrm{g} / \mathrm{ml}$ y como control negativo se empleó solución salina estéril 0,85\% (p/v). Finalmente, las cajas Petri se incubaron a $35 \pm 2{ }^{\circ} \mathrm{C}$ por 24 horas (Alvarado et al., 2010). Los ensayos se realizaron por triplicado.

El porcentaje de inhibición se calculó mediante la ecuación 1, teniendo como referencia el control positivo y la medición del halo de los extractos.

$\%$ Inhibición $=\frac{\phi \text { halo extracto }-\phi \text { halo blanco }}{\phi \text { halo control positivo- } \phi \text { blanco }}$

Análisis estadístico: Se verificó el supuesto de normalidad mediante la prueba de Shapiro Wilk modificada $(p<0,05)$ y se corroboró que no eran normales; por tanto, se evaluó diferencia de efectividad entre los tratamientos con la prueba no paramétrica de Kruskal-Wallis. Los análisis se realizaron usando el software Statistica 8.0. 


\section{Resultados}

Se identificaron las bacterias aisladas en los manipuladores antes del uso de los tratamientos, por medio de tinción de Gram. Los resultados encontrados en los aislamientos pertenecen a bacilos Gram positivos y Gram negativos (Figura 1).

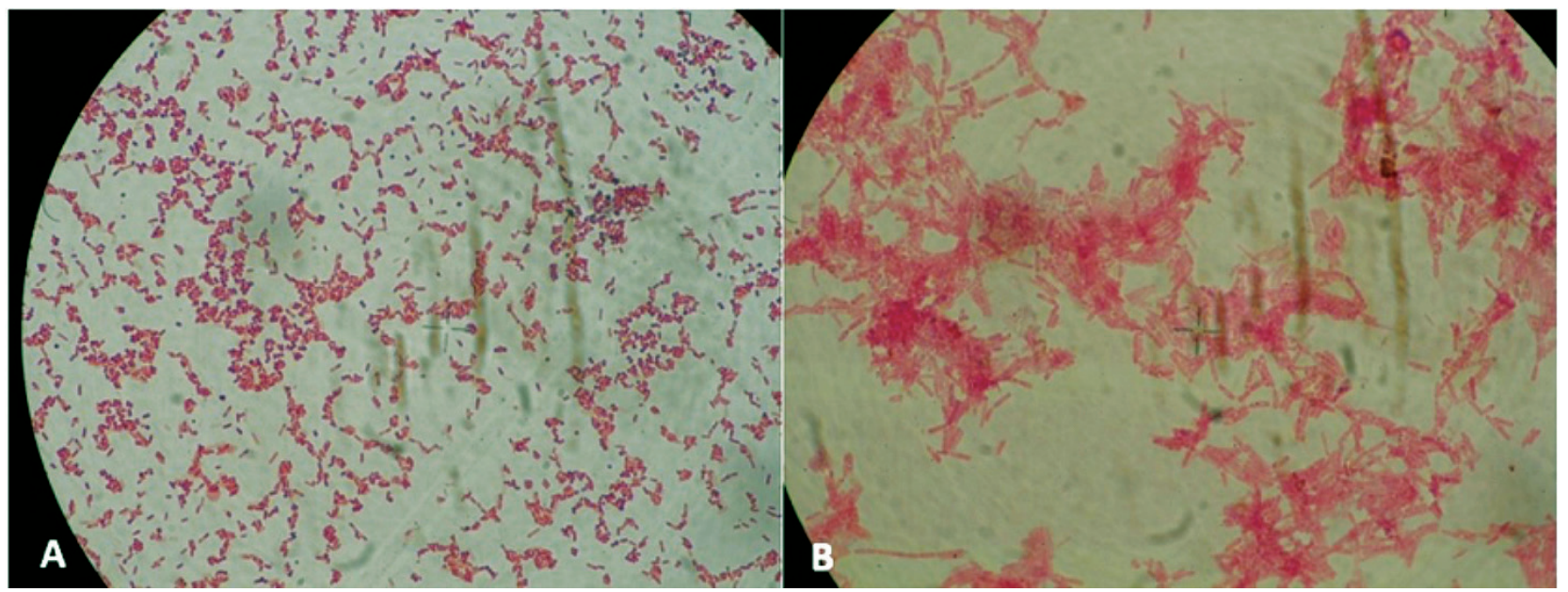

Figura 1. Identificación microscópica de bacterias aisladas a partir de muestreo realizado al personal técnico operativo de la de la Subdirección Científica del Jardín Botánico de Bogotá.

(A) cocos Gram negativos, (B) bacilos Gram negativos.

\section{Efectividad del producto:}

En la Tabla 1 se presenta los datos de los resultados para jabón líquido y sólido.

Tabla 1. Análisis de los datos obtenidos para bacterias.

\begin{tabular}{cccc}
\hline & \multicolumn{3}{c}{ Bacterias } \\
\hline Tratamiento & N & $\begin{array}{c}\text { \% Efectividad } \\
X \pm D P \text { líquido }\end{array}$ & $\begin{array}{c}\text { \% Efectividad } \\
\text { X DP sólido }\end{array}$ \\
\hline A & 21 & $54.31 \pm 48.20^{\mathrm{a}}$ & $21.21 \pm 37.55^{\mathrm{a}}$ \\
B & 21 & $54.39 \pm 48.32^{\mathrm{bc}}$ & $79.26 \pm 35.54^{\mathrm{b}}$ \\
C & 21 & $82.34 \pm 34.49^{\text {cd }}$ & $72 \pm 33^{\mathrm{b}}$ \\
D & 21 & $40.87 \pm 48.50^{\mathrm{abd}}$ & - \\
\hline
\end{tabular}

Letras iguales en la misma columna indican la ausencia de diferencias significativas a través de la prueba de Kruskal-Wallis $(p<0.05)$. N: número de réplicas.

A: Jabón con $0 \%$ de extracto

B: Jabón con $0,1 \%$ de extracto
C: Jabón con $0,2 \%$ de extracto

D: Comercial

El análisis estadístico evidenció que el tratamiento líquido con mayor efectividad fue el de extracto $0,2 \%$, sin embargo, el jabón con $0,1 \%$ de extracto 
y el comercial no son diferentes estadísticamente. Se sugiere usar la concentración $0,1 \%$, puesto que al emplear una menor cantidad de extracto sigue siendo efectivo, aunque no existen diferencias significativas entre los jabones con adición de extracto y el comercial; en este sentido, se estarían reemplazando sustancias sintéticas con el mismo efecto. En estado sólido el tratamiento con $0,1 \%$ de extracto tiene mayor porcentaje de efectividad, aunque no existen diferencias estadísticas entre el tratamiento con $0,1 \%$ y $0,2 \%$ de extracto; se recomienda usar la concentración $0,1 \%$ porque tiene menor cantidad de extracto.

\section{Evaluación actividad antibacteriana frente a cepas de referencia}

En la Tabla 2 se evidencian los resultados cualitativos de la inhibición de los tratamientos frente a las bacterias de referencia (E. coli, S. aureus, S. epidermidis, $P$. aeruginosa). Se representó como (+) la inhibición de los extractos, mientras que las que no presentaron actividad se marcaron como (-).

Tabla 2. Resultados cualitativos de la prueba de actividad antimicrobiana frente a bacterias de referencia.

\begin{tabular}{|c|c|c|c|c|c|c|c|}
\hline Bacteria & L 0 & S 0 & S 0,1 & S 0,2 & L 0,1 & L 0,2 & C \\
\hline $\begin{array}{c}\text { Escherichia coli } \\
\begin{array}{c}\text { Sthaphylococcus } \\
\text { aureus }\end{array}\end{array}(-)$ & $(-)$ & $(-)$ & $(-)$ & $(+)$ & $(+)$ & $(+)$ \\
\hline $\begin{array}{c}\text { Sthaphylococcus } \\
\text { epidermidis }\end{array}$ & $(+)$ & $(+)$ & $(-)$ & $(-)$ & $(-)$ & $(-)$ & $(+)$ \\
\hline $\begin{array}{c}\text { Pseudomonas } \\
\text { aeruginosa }\end{array}$ & $(+)$ & $(-)$ & $(-)$ & $(-)$ & $(+)$ & $(+)$ & $(-)$ \\
\hline
\end{tabular}

L0: líquido con $0 \%$ de extracto; $\mathbf{S} \mathbf{0}$ : sólido con $0 \%$ de extracto; $\mathbf{S} \mathbf{0 , 1}$ : sólido con 0,1 \% de extracto; $\mathbf{S} \mathbf{0 , 2}$ : sólido con $0,2 \%$ de extracto; L $\mathbf{0 , 1}$ : líquido con $0,1 \%$ de extracto; L 0,2: líquido con 0,2\% de extracto; C: jabón comercial en estado líquido.

Los resultados obtenidos demuestran una actividad antibacteriana del jabón líquido con extracto vegetal al $0,1 \%$ y del jabón comercial frente a $E$. coli. Los jabones sólidos y líquidos en todas las concentraciones presentaron inhibición frente a $S$. aureus, incluso el que no tenía extracto funcionó más que el comercial. El jabón elaborado con extracto vegetal de $H$. mexicanum en presentación líquida y con las distintas concentraciones evaluadas tiene actividad inhibitoria frente a los microorganismos estudiados, con un porcentaje mayor que el producto comercial usado como patrón de referencia, confirmando la efectividad de las distintas formulaciones de jabón, convirtiéndolo en una especie promisoria para la bioprospección.

\section{Discusión}

Los resultados de efectividad del producto desinfectante, usando extracto etanólico de $\mathrm{H}$. mexicanum, concuerdan con lo reportado para el género por su actividad (Decosterd, Stoeckli-Evan, Msonthi \& K., 1986). Otras investigaciones reportan actividad antifúngica contra Candida albicans (Rath, Potterat, Mavi \& Hostettmann, 1996), actividad conferida por sustancias etanólicas que se encuentran en la raíz de la planta. El estudio de Rocha et al., (1994) reportó sustancias aisladas del extracto de diclorometano de la corteza y raíz de Hypericum brasiliense con actividad contra Cladosporium cucumericum. Adicionalmente, (Mukherjee, Saritha \& Suresh, 2002) encontraron que los extractos clorofórmico 
y etanólico de las hojas y cortezas de Hypericum patulum e Hypericum mysorense tienen un efecto antifúngico contra hongos de importancia clínica.

Con respecto a la evaluación de la actividad antibacteriana frente a las cepas de referencia, los resultados concuerdan con lo obtenido por Nogueira et al. (2013), quienes evaluaron la actividad antimicrobiana de 15 especies del género Hypericum en Portugal, obteniendo actividad antimicrobiana frente a $S$. aureus. Con respecto a la cepa de $S$. epidermidis, se obtuvo una inhibición del jabón líquido; a diferencia de lo reportado por el estudio de (Dall'Agnol et al., 2003)H. carinatum Griseb., H. connatum Lam., H. ternum A. St. Hil., H. myrianthum Cham. \& Schlecht. and $\mathrm{H}$. polyanthemum Klotzsch ex Reichardt] growing in southern Brazil were analyzed for antimicrobial activity against several microorganisms (bacteria and fungi, en el cual ninguna de las seis especies evaluadas del género Hypericum presentó actividad antibacteriana frente a este microorganismo. Para $P$. aeruginosa se observó inhibición del jabón líquido con concentración de extracto del 0,1\% y $0,2 \%$, resultado congruente con lo encontrado por Sarkisian et al. (2012), quienes probaron siete metabolitos secundarios aislados de las especies Hypericum densiflorum, Hypericum ellipticum, $\mathrm{Hy}$ pericum prolificum, y Hypericum punctatum como inhibidores de crecimiento de bacterias como Staphylococcus spp., Escherichia coli y Pseudomonas aeruginosa; estos resultados concuerdan con el estudio de Gibbons et al. (2002), en el cual se indica que el género Hypericum tiene un gran potencial para producir compuestos antibacterianos.

Adicionalmente, los resultados obtenidos en esta investigación concuerdan con la evaluación de la actividad antibacteriana realizada por Rodríguez (2012) y, de la misma manera, ratifica el uso etnobotánico reportado. Por lo tanto, se puede decir que la especie presenta potencial antibacteriano, el cual se sugiere seguir estudiando con el fin de identificar la fracción responsable de la actividad antibacteriana, esto puede permitir que la especie se emplee como ingrediente bioactivo. Se concluye que los jabones formulados con extracto vegetal de Hypericum mexicanum $\mathrm{L}$. tienen actividad antibacteriana frente a microorganismos de importancia en salud pública como $E$. coli, S. aureus, S. epidermidis y $P$. aeruginosa. Dando resultado al objetivo del presente trabajo y ampliando el potencial investigativo del género Hypericum.

\section{Agradecimientos}

Las autoras expresan su agradecimiento al Jardín Botánico de Bogotá José Celestino Mutis, centro de investigación y desarrollo científico, por el apoyo para la realización de esta investigación; a Erika Andrea Plazas, por la realización del extracto crudo; a las personas de la Subdirección Científica que colaboraron con la prueba de manipuladores y a Edna Arévalo y Johana Romero, por su apoyo en el análisis estadístico.

\section{Literatura citada}

1. Alvarado, D., García, J. D. \& Arias-echandi, M. L. (2010). Manos y su estabilidad a través del tiempo, 21(1), 29-31.

2. Barnes, J., Anderson, L. A. \& Phillipson, J. D. (2001). St John's wort (Hypericum perforatum L.): a review of its chemistry, pharmacology and clinical properties. Journal of Pharmacy and Pharmacology, 53(5), 583-600. https://doi.org/10.1211/0022357011775910

3. Castro, C. (2011). Manual de toma de muestras. Unidad clínica de enfermedades infecciosas y microbiología, 112. Recuperado de: http://ahvalme.org/ RepositorioDocman/ugc/infecciosos/Manual_Toma_ Muestras_Microbiologia.pdf

4. Dall'Agnol, R., Ferraz, a, Bernardi, a P., Albring, D., Nör, C., Sarmento, L.,... \& Schapoval, E. E. S. (2003). Antimicrobial activity of some Hypericum species. Phytomedicine: International Journal of Phytotherapy and Phytopharmacology, 10, 511-516. https://doi. org/10.1078/094471103322331476

5. Decosterd, L., Stoeckli-Evan, H., Msonthi, J.D., H. \& K. (1986). A new antifungal chromene and a related dichromene from Hypericum revolutum. Planta Médica, 55, 429.

6. Gibbons, S. (2008). Phytochemicals for bacterial resistance--strengths, weaknesses and opportunities. Planta Médica, 74(6), 594-602. https://doi. org/10.1055/s-2008-1074518

7. Gibbons, S., Ohlendorf, B., \& Johnsen, I. (2002). The genus Hypericum - A valuable resource of anti-Staphylococcal leads. Fitoterapia, 73 (4), 300-304. https:// doi.org/10.1016/S0367-326X(02)00082-5 
8. Gutiérrez, M., Pineda, M. \& García, A. (2011). Las maticas de mi región. In Jardín Botánico de Bogotá José Celestino Mutis. (p. 33).

9. Ishiguro, K., Yamaki, M., Kashihara, M. \& Takagi, S. (1986). Sarothralen A and B, new antibiotic compounds from Hypericum japonicum. Planta Médica, 52 (Table II), 288-90. https://doi.org/10.1055/s-2007-969154

10. Jayasuriya $H$, McChesney JD, Swanson SM, P. J. (1989). Antimicrobial and cytotoxic activity of rottlerintype compounds from Hypericum drummondii. Journal of Natural Products, 52(2), 325-331

11. Melgarejo, L. M., J. Sánchez, A. Chaparro, F. NEWMARK, M. Santos-Acevedo, C. Burbano \& Reyes, C. (2002). Aproximación al estado actual de la bioprospección en Colombia Bogotá: Cargraphics 334p.--(Serie de Documentos Generales INVEMAR No.10). Recuperado de: http://www.invemar.org.co/redcostera1/invemar/docs/ ESTADO_BIOPROSPECCION.pdf

12. Mukherjee, P. K., Saritha, G. S. \& Suresh, B. (2002). Antimicrobial potential of two different Hypericum species available in India. Phytotherapy Research, 16(7), 692-695. https://doi.org/10.1002/ptr.1016

13. Newman, D. J., \& Cragg, G. M. (2007). Natural products as sources of new drugs over the last 25 years. Journal of Natural Products. https://doi.org/10.1021/np068054v

14. Nogueira, T., Medeiros, M. A., Marcelo-Curto, M. J., García-Pérez, B. E., Luna-Herrera, J. \& Costa, M. C. (2013). Profile of antimicrobial potential of fifteen Hypericum species from Portugal. Industrial Crops and Products, 47, 126-131. https://doi.org/10.1016/j.indcrop.2013.03.005

15. Pistelli, L., Bertoli, A., Zucconelli, S., Morelli, I., Panizzi, L. \& Menichini, F. (2000). Antimicrobial activity of crude extracts and pure compounds of Hypericum hircinum. Fitoterapia, 71(SUPPL. 1). https://doi.org/10.1016/ S0367-326X(00)00189-1

16. Rabanal, R. ., Arias, A., Prado, B., Hernández-Pérez, M. \& Sánchez-Mateo, C. . (2002). Antimicrobial studies on three species of Hypericum from the Canary Islands. Journal of Ethnopharmacology, 81(2), 287-292. https://doi.org/10.1016/S0378-8741(02)00083-1

17. Rath, G., Potterat, O., Mavi, S. \& Hostettmann, K. (1996). Xanthones from Hypericum roeperanum. Phytochemistry, 43(2), 513-520. https://doi.org/10.1016/00319422(96)00284-1

18. Rocha, L., Marston, A., Auxiliadora, M., Kaplan, C., Stoeckli-Evans, H., Thull, U., ... Hostettmann, K. (1994). An antifungal gamma-pyrone and xanthones with monoamine oxidase inhibitory activity from Hypericum brasiliense. Phytochemistry, 36(6), 1381-1385. https://doi.org/10.1016/S0031-9422(00)89727-7

19. Rodríguez, V. (2012). Estudio fitoquímico preliminar y evaluación de la actividad antibacteriana in vitro de extractos y fracciones de la especie Hypericum mexicanum. Universidad del Bosque.

20. Sarkisian, S. A., Janssen, M. J., Matta, H., Henry, G. E., Laplante, K. L., \& Rowley, D. C. (2012). Inhibition of bacterial growth and biofilm production by constituents from Hypericum spp. Phytotherapy Research, 26(7), 1012-1016. https://doi.org/10.1002/ptr.3675

21. Sterman, C. (2001). Jabones Esenciales. (Cartoné $Y$ Color, Ed.).

22. Yamaki, M. \& Ishiguro, K. (1994). Antimicrobial activity of naturally occurring and synthetic phloroglucinol against Staphylococcus aureus. Phytotherapy Research, $8(2), 112-114$.
Conflicto de Intereses Los autores declaran no tener ningún conflicto de intereses

Recibido: septiembre 07 de 2016 Aceptado: octubre 20 de 2016 